EPR studies of the dimeric compound $\mathrm{Cu}($ tda)phen and its $\mathrm{Zn}(\mathrm{II})$ isostructural analog doped with $\mathrm{Cu}(\mathrm{II})$ allowed us to determine the magnetic parameters of the $\mathrm{Cu}(\mathrm{II})$ ions which compose the dimeric units, as well as the magnetic interaction between them. Analysis of the single-crystal spectra in the magnetically concentrated system using Anderson's exchange narrowing model, extendend by us to consider dimer populations with neighbors in different magnetic states, allowed us to determine the magnitude of interdimeric interactions associated with $\pi-\pi$ interactions between phenanthroline ligands.

Studies of $\mathrm{Co}(\mathrm{II})$ Fumarato and $\mathrm{Zn}(\mathrm{II}) \mathrm{Co}(\mathrm{II})$
Fumarato allowed us to determine the magnetic parameters associated with Co(II) ions in a distorted octahedral environment. Analysis of magnetic measurements and EPR results in magnetically concentrated and diluted samples allowed us to determine magnetic interactions between Co(II) ions associated with chemical paths which involve Hydrogen bonds or the fumarate anion ligand. Also we established correlations between magnetic parameters and the coordination environment, which we interpreted performing calculations based on the crystal field theory, and between magnetic couplings and the chemical paths which connect the metallic ions.

\title{
Implicancias de la arquitectura en la regeneración vegetativa de tres malezas perennes tolerantes a glifosato con diferentes formas de crecimiento
}

\section{Elisa Panigo \\ epanigo@fca.unl.edu.ar \\ Dra. Mariel G. Perreta \\ Dr. Abelardo C. Vegetti \\ Cátedra de Morfología Vegetal \\ Facultad de Ciencias Agrarias \\ Universidad Nacional del Litoral \\ Fecha de la defensa: 26/03/2013}

\section{Resumen}

El objetivo fue identificar los caracteres morfológicos que facilitan la regeneración vegetativa de tres malezas tolerantes a glifosato: Commelina erecta L., Eustachys retusa Lag. Kunth. y Alternanthera philoxeroides Mart. Griseb., en los sistemas de producción con mínima labranza y uso intensivo de glifosato; y predecir su comportamiento. Primero se determinó la arqui- tectura del sistema de vástagos aéreos de las tres especies y su evolución desde la germinación. Luego se evaluaron las variaciones morfológicas, de crecimiento, bioquímicas y arquitecturales post-aplicación de glifosato en las tres especies, para identificar las estrategias que facilitan su regeneración y persistencia a campo. Se trabajó con 2 dosis de herbicida (letal y subletal) en cada especie. Debido a los diferentes niveles de susceptibilidad al herbicida que presentan estas tres malezas, las dosis para cada especie se seleccionaron a partir de un ensayo de dosis-respuesta. Por último, se analizó la presencia y ubicación de zonas meristemáticas de $C$. erecta y $E$. retusa para evaluar el rol que juegan en la regeneración vegetativa post-aplicación.

A partir del análisis arquitectural se iden- 
tificaron distintos caracteres comunes que podrían tener implicancias en la regeneración vegetativa ante situaciones de estrés, como: 1) la presencia de un tallo reservante con yemas axilares normalmente inhibidas, 2) una unidad arquitectural, que se establece durante la primera estación de crecimiento, compuesta por pocas categorías de ejes que son independientes del orden de ramificación y 3) ramificaciones basales que alcanzan un gran desarrollo y poseen una estructura semejante a su parental y presencia de reiteración.

El comportamiento frente a glifosato determinó una serie de respuestas comunes y otras particulares de cada especie. A nivel general, se afectó la altura de los ejes, el número de ramas e inflorescencias, la biomasa, la concentración de clorofila y la superficie foliar. Sin embargo, la magnitud del efecto dependió de la dosis y la especie. A nivel particular se observó que $C$. erecta mostró dos fenotipos de respuesta, con variaciones en la zona de producción de ramificaciones. En E. retusa aumentó el orden de ramificación de las plantas de manera significativa con respecto al control. A. philoxeroides mostró una baja tolerancia al herbicida que causó el deterioro de la mayor parte de los órganos aéreos, lo que podría estar relacionado con las condiciones de evaluación más que con una alta susceptibilidad.

Con respecto a las zonas meristemáticas, $C$. erecta y $E$. retusa mantuvieron un importante banco de yemas latentes y viables, en la zona basal y media de los ejes post-aplicación de glifosato, por lo que el herbicida no limita la capacidad de rebrotar de estas especies.

A través de los resultados obtenidos, integrando los estudios estructurales con las características de crecimiento y bioquímicas, se han podido reconocer un conjunto de caracteres morfológicos comunes, en tres especies con formas de crecimiento completamente diferente. Estos caracteres juegan un rol importante en el escape a la acción del herbicida, y parecen en cierta medida independientes de las especies, y se relacionan más con la arquitectura de la planta.

\section{Implications of architecture in vegetative regeneration of perennial glyphosate- \\ tolerant weeds}

\section{Summary}

The objective was to identify morphological traits involved in the vegetative regeneration of Commelina erecta L., Eustachys retusa Lag. Kunth. and Alternanthera philoxeroides Mart. Griseb., in productive systems with intensive use of glyphosate, and to predict their response.

Through architectural analysis, several traits that enable regeneration were identified: The presence of a storage stem with axillary buds bank, an architectural unity established during the first growing season characterized by a few axis categories basal ramifications that reach a great development and resemble their parental structure, and the presence of reiteration. In order to evaluate the response to glyphosate application, different doses were selected from a doses-response assay. In general, the herbicide affected negatively the height, number of branches and inflorescences, as well as biomass, chlorophyll content, and leaf surface of the three species. In particular, C. erecta showed two phenotypes differing in the zone that produces the branches. $E$. retusa had no difference in the position of branches, but experienced an increase in the order of branching. A. philoxeroides showed low tolerance to the herbicide. 
The meristematic zones of $C$. erecta y $E$. retusa, kept an important bank of dormant and viable buds, in the basal and medial zone of the axis after herbicide application, which means that the glyphosate do not reduce the ability of regrowth in these species.

According to these results, several morphological characters shared by the three species were recognized, which may play an important role in avoiding the herbicide action.

\section{Caracterización molecular de integrones clase 1. Expresión y caracterización funcional de orfs asociados}

\author{
Ayelén Patricia Porto \\ ayelenporto1@gmail.com \\ Dr. Gabriel Gutkind / Dr. José Di Conza \\ Cátedra de Microbiología General \\ Facultad de Bioquímica y Cs Biológicas \\ Universidad Nacional del Litoral \\ Fecha de la defensa: 18/03/2013
}

\section{Resumen}

La diseminación de determinantes de resistencia a antimicrobianos a través del intercambio de material genético entre las poblaciones bacterianas, y la posterior emergencia de microorganismos resistentes es un fenómeno actual y en crecimiento. Uno de los elementos involucrados en la integración y expresión de genes de resistencia a antimicrobianos son los integrones. Estos son elementos genéticos móviles que actúan como vectores naturales de clonado y expresión integrando y expresando genes en casete.

Como parte de un estudio epidemiológico de integrones realizado con anterioridad en nuestra región, se ha detectado en tres aislamientos multiresistentes de enterobacterias un nuevo gen en casete codificante para una $\beta$-lactamasa ( bla $_{\text {OXA-101 }}$ ) derivada de bla ${ }_{\text {OXA-10 }}$ localizado en un integrón clase 1. Las $\beta$-lactamasas de tipo OXA han sido descriptas en enterobacterias y en otros bacilos gram-negativos no fermentadores de glucosa (principalmente en Acinetobacter baumanii y Pseudomonas aeruginosa). Se hallan frecuentemente localizadas en plásmidos e integrones, asiduamente como genes en casete dentro de integrones clase 1.

Con relación a este tema, en éste trabajo de tesis se planteó el análisis de la localización y transferibilidad genética de bla ${ }_{\mathrm{OXA}-101}$, y el estudio de las características bioquímicas de la $\beta$-lactamasa. Los resultados obtenidos permiten afirmar que este gen en casete se encuentra localizado en un plásmido conjugativo de alto peso molecular. Se determinó además, el punto isoeléctrico y masa molecular de la enzima obtenida de forma recombinante. Respecto al espectro de hidrólisis, sólo se observó una disminución de la susceptibilidad a ampicilina y piperacilina netamente debido a OXA-101. Por último, se realizó una breve caracterización cinética de la misma.

Dentro de los integrones clase 1, existen los denominados integrones inusuales. Estos poseen como característica única la presencia de una región común (CR), que contiene el marco abierto de lectura orf513, codificante para una posible recombinasa de ADN. Se encuentra postulado que la proteína Orf513 podría estar involucrada 